\title{
BOUNDARY ELEMENT METHOD ANALYSIS OF BOUNDARY VALUE PROBLEMS WITH PERIODIC BOUNDARY CONDITIONS
}

\author{
VASYL I. GNITKO ${ }^{1}$, ARTEM O. KARAIEV ${ }^{2 *}$, NEELAM CHOUDHARY ${ }^{3}$ \& ELENA A. STRELNIKOVA ${ }^{1,2 \dagger}$ \\ ${ }^{1}$ A. Podgorny Institute of Mechanical Engineering Problems of the Ukrainian Academy of Sciences, Ukraine \\ ${ }^{2}$ V.N. Karazin Kharkiv National University, Ukraine \\ ${ }^{3}$ Bennett University, India
}

\begin{abstract}
This paper explores non-axisymmetric boundary value problems for the Laplace equation. Neumann's, Dirichlet's and mixed boundary conditions are involved, supposing their periodic behaviour. Boundary value problems arise as auxiliary issues in many practical applications. Among them there are problems related to numerical simulation of vibrations of fluid-filled elastic shells of revolution, coupled vibrations of elastic circular plates resting on a sloshing liquid, crack propagation in elastic mediums, and more. The common feature in these problems is the necessity to obtain the numerical solution of the Laplace equation under different boundary conditions. As these problems are auxiliary, it is necessary to obtain their numerical solutions with high accuracy. The most effective method to solve these problems is the boundary elements method (BEM). Here a new variant of BEM is proposed for the axisymmetric calculation domain with given periodic functions for boundary conditions. The shape of the calculation domain allows us to reduce surface integral equations to one-dimensional ones. In doing so, we must evaluate elliptic-like inner integrals with high accuracy, to elaborate the method of calculation of the outer integrals with logarithmic, Cauchy or Hadamard finite part singularities. An efficient method for evaluating elliptic-like integrals was developed using a special series for integrands, and the quadrature equations were obtained for highprecision calculation of outer integrals. The method developed can be used to determine free vibration modes and frequencies for elastic fluid-filled shells of revolution.
\end{abstract}

Keywords: boundary element method, periodic boundary conditions, singular integral equations, free vibrations, fluid-filled elastic shells.

\section{INTRODUCTION}

Mixed boundary value problems for elliptic equations with periodic boundary conditions occur in a wide range of engineering applications, such as composite and fluid mechanics [1], [2], vibrations of structural elements [3], crack propagation in elastic mediums [4], fluid-structure interactions [5], [6], cyclically symmetrical structure design [7], and more.

In modelling mechanical processes, systems of differential equations with periodic boundary value conditions are usually involved. Analytical solutions of these equations have been obtained for some simple cases, but advanced numerical methods are currently needed in mechanical and engineering applications [8], [9]. For successive applications of numerical methods to solve periodic boundary value problems (PBVP), theorems are needed regarding periodic behaviour of the solutions. Some of these theorems were proven [10], [11]. The existing theorems allow us to use Fourier series coupled with the finite element method (FEM), finite difference method (FDM) or boundary element method (BEM), widely used in PBVP for differential equations. In using FEM and FDM, the main obstacle is mesh-generating for an arbitrary domain that requires large amounts of

* ORCID: https://orcid.org/0000-0003-3176-8496

$\dagger$ ORCID: http://orcid.org/0000-0003-0707-7214 
processing power and computation time. In recent decades, the BEM originated and developed by Carlos Brebbia [12] has become increasingly popular, due to its unique feature of mesh reduction.

In this paper, the non-axisymmetric periodic boundary value problems of free liquid surface vibrations in shells of revolution and elastic shell vibrations are formulated, and then solved using BEM coupled with Fourier series.

\section{NON-AXISYMMETRIC PERIODIC BOUNDARY VALUE PROBLEMS OF SHELL VIBRATIONS}

\subsection{Problem statement}

Consider a coupled problem of dynamic behaviour of an elastic shell of revolution partially filled with a liquid. Such shells can be used as models for numerical simulation of liquid disturbance/sloshing and vibrations of the shell walls in fuel tanks, oil storage reservoirs, water storage containers, etc. The wet part of the shell surface is denoted as $S_{1}$ and the liquid free surface as $S_{0}$ (Fig. 1).
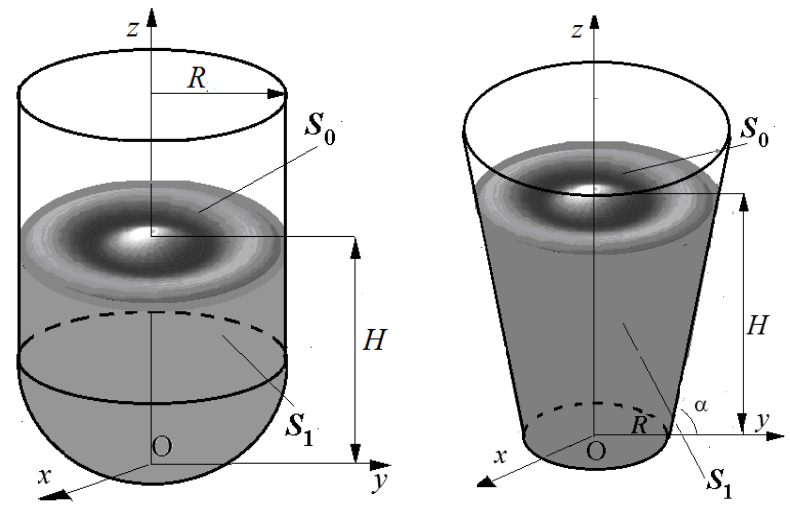

Figure 1: Shells of revolution partially filled with a liquid.

For a liquid that is flowing, we consider two conservation laws: conservation of mass and conservation of momentum. Letting $\mathbf{V}$ be the velocity field of the fluid, $\rho$ the liquid density, the continuity equation (law of mass conservation) in the absence of mass sources is (eqn 1):

$$
\frac{\partial \rho}{\partial t}+\operatorname{div}(\rho \mathbf{V})=0
$$

The momentum conservation law states that the rate of momentum change is equal to the applied forces. Forces acting on the liquid control volume are the integral of the stress tensor, $\sigma_{i j}$, over the surface; plus the integral of body force vectors per unit mass, over the volume. Considering the motion of a continuous, viscous fluid, the stress in the fluid is composed of two parts; a locally isotropic part proportional to the scalar pressure field and a non-isotropic part, due to viscous friction. The stress tensor follows eqn (2); 


$$
\sigma_{i j}=-p \delta_{i j}+\tau_{i j}
$$

where $p$ is the pressure, $\delta_{i j}$ is the Kronecker unit tensor, and $\tau_{i j}$ is the viscous stress tensor.

Suppose that the liquid inside the shell is an ideal $\left(\tau_{i j}=0\right)$ one, and the surface tension is neglected: We then have the following eqn (3) for motion under the force of gravity $\rho \mathbf{g}$ :

$$
\rho\left(\frac{\partial \mathbf{V}}{\partial t}+(\mathbf{V} \cdot \nabla) \mathbf{V}\right)=-\nabla p-\rho \nabla(g z)
$$

For incompressible inhomogeneous liquids, conservation laws in linear formulation became:

$$
\begin{gathered}
\operatorname{div}(\mathbf{V})=0, \\
\rho \frac{\partial \mathbf{V}}{\partial t}=-\nabla p-\rho \nabla(g z) .
\end{gathered}
$$

If the fluid flow is not rotational ( $\operatorname{rot} \mathbf{V}=0$ ), then the potential flow theory could be applied. Thus, a scalar velocity potential of $\Phi=\Phi(x, y, z, t)$ can be introduced, with $\mathbf{V}=\nabla \Phi$. Then eqn (1) is transformed to the Laplace equation:

$$
\Delta \Phi=0 \text {. }
$$

From momentum conservation law (2) we obtain:

$$
p-p_{0}=-\rho\left(\frac{\partial \Phi}{\partial t}+g z\right)
$$

where $p_{0}$ is atmospheric pressure.

To determine the potential $\Phi$, a mixed boundary value problem for the Laplace equation was formulated. The non-penetration condition of the wet tank surface $\left(S_{1}\right)$ was applied [13]. On the free surface, the following kinematic and dynamic boundary conditions are satisfied [6], [9]:

$$
\left.\frac{\partial \Phi}{\partial n}\right|_{S_{0}}=\frac{\partial \zeta}{\partial t}, \quad p-\left.p_{0}\right|_{S_{0}}=0
$$

Here, an unknown function $\zeta=\zeta(x, y, t)$ is the free surface elevation measured vertically, above the still water level.

This function describes the shape and position of the free surface. Thus, the following boundary value problem was formulated:

$$
\Delta \Phi=0,\left.\quad \frac{\partial \Phi}{\partial n}\right|_{S_{1}}=0,\left.\quad \frac{\partial \Phi}{\partial n}\right|_{S_{0}}=\frac{\partial \zeta}{\partial t}, \quad p-\left.p_{0}\right|_{S_{0}}=0
$$

where $p-p_{0}$ is received from eqn (4) at $z=\zeta(x, y, t)$, and $n$ is an external unit normal to the corresponding surfaces.

The solvability condition for the Neumann problem (6) is given [13]: 


$$
\iint_{S_{0}} \frac{\partial \Phi}{\partial n} d S_{0}=0
$$

The boundary value problem (6), (7), was formulated for studying the liquid vibrations within the shell. Problems involving axisymmetric bodies with arbitrary boundary conditions are best treated using cylindrical coordinates. With a cylindrical coordinates system $(\rho, z, \alpha)$, one can represent the velocity potential $\Phi$ that satisfies a given boundary value problem (6), (7), as follows [6]:

$$
\Phi=\sum_{l=0}^{N} \sum_{k=1}^{M} \dot{d}_{l k}(t) \varphi_{l k}(\rho, z, \alpha)
$$

From eqns (6) and (8), the next presentation is obtained for determining the free surface elevation $\zeta$ :

$$
\zeta=\frac{1}{g} \sum_{l=0}^{N} \sum_{k=1}^{M} d_{l k}(t) \zeta_{l k}(\rho, 0, \alpha) .
$$

Here, $d_{l k}(t)$ are unknown time-dependent functions, while the $\varphi_{l k}$ functions satisfy the following equations:

$$
\Delta \varphi_{l k}=0,\left.\quad \frac{\partial \varphi_{l k}}{\partial n}\right|_{S_{1}}=0,\left.\quad \frac{\partial \varphi_{l k}}{\partial n}\right|_{S_{0}}=\zeta_{l k} .
$$

Considering fluid-filled elastic shells without including the force of gravity [5], we arrive at a similar problem. Namely, the solution for the potential $\Phi$ in the following equation is in use:

$$
\Phi=\sum_{l=0}^{N} \sum_{k=1}^{M} \dot{c}_{l k}(t) \psi_{l k}(\rho, z, \alpha) .
$$

Here, $c_{l k}(t)$ are unknown time-dependent functions, while functions for $\psi_{l k}$ are obtained from the following boundary value problems [5]:

$$
\Delta \psi_{l k}=0,\left.\quad \frac{\partial \psi_{l k}}{\partial n}\right|_{S_{1}}=u_{l k},\left.\quad \psi_{l k}\right|_{S_{0}}=0,
$$

where the $u_{l k}$ functions are their own modes of the empty elastic shell vibrations.

If the fluid-filled shell being considered is a shell of revolution, then dependency of the angular coordinate for unknown functions can be most conveniently described by Fourier series. Therefore, for the $\zeta_{l k}$ and $u_{l k}$ functions, the following equations are used:

$$
\zeta_{l k}(\rho, 0, \alpha)=\widetilde{\zeta}_{l k}(\rho) \cos l \alpha, \quad u_{l k}(\rho, z, \alpha)=\widetilde{u}_{l k}(\rho, z) \cos l \alpha .
$$

Then, discretisation of the body requires only meshing the shell generator with onedimensional boundary elements, which substantially decreases the number of unknowns. The drawback of this method is in the increasing complexity of numerical implementation, 
because integrals over the angle for shells of revolution must be calculated for every Fourier term. Herein, we have elaborated this effective method to calculate the angular integrals.

\subsection{Periodic boundary value problems}

Without loss of generality, the periodic boundary value problem statement in cylindrical coordinates can be expressed in the following form:

$$
\Delta u=0,\left.\quad \frac{\partial u}{\partial n}\right|_{S_{1}}=f(\rho, z) \cos (l \alpha),\left.\quad u\right|_{S_{0}}=0 .
$$

Here, $u$ is an unknown function, $\Delta$ is the Laplace operator, $S_{0}$ is the free surface (of liquid), $S_{1}$ is for the inside shell surfaces, $f(\rho, z)$ is a known boundary function, and $l$ is an integer number.

It should be noted that the boundary conditions in problems (10)-(12) are nonaxisymmetric. So we need to use this property in integral representations of the problems under consideration. To solve the Laplace equation, we used the well-known third Green's identity, which represents solutions for the Laplace equation in the following integral form:

$$
2 \pi u(\xi)+\oint_{S} q^{*}(\xi, \mathbf{r}) u(\mathbf{r}) d S(\mathbf{r})=\oint_{S} u^{*}(\xi, \mathbf{r}) \frac{\partial u}{\partial n}(\mathbf{r}) d S(\mathbf{r}) .
$$

Here, $u^{*}(\xi, \mathbf{r})$ and $q^{*}(\xi, \mathbf{r})$ are expressed by Green's function of the Laplace equation and its normal derivative, and $S=S_{0} \cup S_{1}$.

Hereinafter, we will suppose that the body's boundary is axisymmetric with nonaxisymmetric boundary conditions; therefore, we can make a transformation from surface integral to contour in the following manner, where $\Gamma$ is a generator of the surface $S$ :

$$
2 \pi u(\xi)+\oint_{\Gamma} \rho d \Gamma(\mathbf{r}) \int_{0}^{2 \pi} q^{*}(\xi, \mathbf{r}) u(\mathbf{r}) d \alpha=\oint_{\Gamma} \rho d \Gamma(\mathbf{r}) \int_{0}^{2 \pi} u^{*}(\xi, \mathbf{r}) \frac{\partial u}{\partial n}(\mathbf{r}) d \alpha .
$$

In this study, due to the boundary conditions in eqn (12), we can present the unknown function in the following form:

$$
u(\mathbf{r})=u(\rho, z) \cos (l \alpha)
$$

Here $u(\rho, z)$ is an unknown axisymmetric function, yet non-axisymmetric behaviour within the problem is taken into account by multiplying this function by cosines, so that eqn (13) takes the form of:

$$
\begin{aligned}
2 \pi u(\xi)+\int_{\Gamma_{1}} u(\rho, z) \rho d \Gamma(\mathbf{r}) \int_{0}^{2 \pi} q^{*}(\xi, \mathbf{r}) \cos (l \alpha) d \alpha=\int_{\Gamma_{0}} \frac{\partial u}{\partial n}(\rho, z) p d \Gamma(\mathbf{r}) \int_{0}^{2 \pi} u^{*}(\xi, \mathbf{r}) \cos (l \alpha) d \alpha+ \\
+\int_{\Gamma_{1}} f(\rho, z) p d \Gamma(\mathbf{r}) \int_{0}^{2 \pi} u^{*}(\xi, \mathbf{r}) \cos (l \alpha) d \alpha
\end{aligned}
$$


Here, $\Gamma=\Gamma_{0} \cup \Gamma_{1}, \Gamma_{0}$ and $\Gamma_{1}$ are generators of the surfaces $S_{0}$ and $S_{1}$, respectively. So the topical issue addressed here is to calculate axial integrals with Green's functions, multiplied by trigonometric functions.

2.3 Axial integrals with Green's functions and trigonometric functions

It is necessary to find a way of estimating the next integrals:

$$
I_{u}\left(\rho, \rho_{0}, z, z_{0}\right)=\int_{0}^{2 \pi} u^{*}(\xi, \mathbf{r}) \cos (l \alpha) d \alpha, \quad I_{q}\left(\rho, \rho_{0}, z, z_{0}\right)=\int_{0}^{2 \pi} q^{*}(\xi, \mathbf{r}) \cos (l \alpha) d \alpha .
$$

Here, the following Green's functions are involved:

$$
u^{*}(\xi, \mathbf{r})=\frac{1}{|\xi-\mathbf{r}|}, \quad q^{*}(\xi, \mathbf{r})=\frac{(\xi-\mathbf{r}, \mathbf{n}(\mathbf{r}))}{|\xi-\mathbf{r}|^{3}} .
$$

By using the expressions described in eqn (15), one can obtain $I_{u}\left(\rho, \rho_{0}, z, z_{0}\right)$ as seen in the next expression:

$$
\int_{0}^{2 \pi} u^{*}(\xi, \mathbf{r}) \cos (l \alpha) d \alpha=\int_{0}^{2 \pi} \frac{\cos (l \alpha) d\left(\alpha-\alpha_{0}\right)}{\sqrt{\rho^{2}+\rho_{0}^{2}+\left(z-z_{0}\right)^{2}-2 \rho \rho_{0} \cos \left(\alpha-\alpha_{0}\right)}} .
$$

Then, with substitution of:

$$
\theta=\frac{\alpha-\alpha_{0}-\pi}{2} \Rightarrow \alpha-\alpha_{0}=2 \theta+\pi
$$

the integral $I_{u}\left(\rho, \rho_{0}, z, z_{0}\right)$ transforms into the following:

$$
I_{u}\left(\rho, \rho_{0}, z, z_{0}\right)=\frac{4(-1)^{l} \cos \left(l \alpha_{0}\right)}{\sqrt{\left(\rho+\rho_{0}\right)^{2}+\left(z-z_{0}\right)^{2}}} \int_{0}^{\frac{\pi}{2}} \frac{\cos (2 l \theta) d \theta}{\sqrt{1-m^{2} \sin ^{2} \theta}}, m^{2}=\frac{4 \rho \rho_{0}}{\left(\rho+\rho_{0}\right)^{2}+\left(z-z_{0}\right)^{2}} .
$$

To calculate this integral, we need to expand the cosine functions into a series. This procedure can be accomplished by using De Moivre's equation:

$$
\begin{aligned}
& \cos (2 l \theta)=\operatorname{Re}\left[e^{2 i l \theta}\right]=\operatorname{Re}(\cos (2 l \theta)+i \sin (2 l \theta))=\operatorname{Re}(\cos \theta+i \sin \theta)^{2 l}= \\
& =\operatorname{Re} \sum_{k=0}^{2 l} C_{2 l}^{k} \cos ^{2 l-k} 2 \theta \sin ^{k} 2 \theta(i)^{k}=\sum_{k=0}^{2 l} C_{2 l}^{k} \cos ^{2 l-k} 2 \theta \sin ^{k} 2 \theta \operatorname{Re}(i)^{k} .
\end{aligned}
$$

It should be noted that $\operatorname{Re}(i)^{k}=0, \forall k=2 n+1$.

Thus, by changing the summation indexes, it is possible to obtain: 


$$
\begin{gathered}
\sum_{k=0}^{2 l} C_{2 l}^{k} \cos ^{2 l-k} 2 \theta \sin ^{k} 2 \theta \operatorname{Re}(i)^{k}=\left|\begin{array}{c}
2 p=k \\
k=0 \Rightarrow p=0, k=2 l \Rightarrow p=l
\end{array}\right|= \\
=\sum_{p=0}^{l}(-1)^{p} C_{2 l}^{2 p} \cos ^{2(l-p)} \theta \sin ^{2 p} \theta=\sum_{p=0}^{l}(-1)^{p} C_{2 l}^{2 p}\left(1-\sin ^{2} \theta\right)^{l-p} \sin ^{2 p} \theta= \\
=\sum_{p=0}^{l}(-1)^{p} C_{2 l}^{2 p} \sin ^{2 p} \theta \sum_{k=0}^{l-p} C_{l-p}^{k}(-1)^{k} \sin ^{2 k} \theta=\left|\begin{array}{c}
s=p+k \\
k=0 \Rightarrow s=p \\
k=l-p \Rightarrow s=l
\end{array}\right|= \\
=\sum_{p=0}^{l} \sum_{s=p}^{l}(-1)^{s} C_{2 l}^{2 p} C_{l-p}^{l-s} \sin ^{2 s} \theta
\end{gathered} .
$$

If we then add the term $\sum_{s=0}^{p-1}(-1)^{s} C_{2 l}^{2 p} C_{l-p}^{l-s} \sin ^{2 s} \theta$ into the inner sum, the above result does not change due to the properties of the Euler's gamma function. We can therefore get an expression with independent sum indexes:

$$
\begin{gathered}
\sum_{p=0}^{l} \sum_{s=p}^{l}(-1)^{s} C_{2 l}^{2 p} C_{l-p}^{l-s} \sin ^{2 s} \theta=\sum_{p=0}^{l} \sum_{s=0}^{l}(-1)^{s} C_{2 l}^{2 p} C_{l-p}^{l-s} \sin ^{2 s} \theta= \\
=\sum_{s=0}^{l}(-1)^{s} \sin ^{2 s} \theta \sum_{p=0}^{l} C_{2 l}^{2 p} C_{l-p}^{l-s} .
\end{gathered}
$$

The inner sum can be calculated using the Yegorychev summation method [14]:

$$
\sum_{p=0}^{l} C_{2 l}^{2 p} C_{l-p}^{l-s}=\sum_{p=0}^{s} C_{2 l}^{2 p} C_{l-p}^{l-s}=\frac{4^{s} l}{l+s} C_{l+s}^{2 s} .
$$

Thus, the double cosine function has the following presentation:

$$
\cos (2 l \theta)=\sum_{s=0}^{l} \frac{4^{s}(-1)^{s} l}{l+s} C_{l+s}^{2 s} \sin ^{2 s} \theta
$$

Using expansion in eqn (16), the integral $I_{u}\left(\rho, \rho_{0}, z, z_{0}\right)$ was transformed into:

$$
I_{u}\left(\rho, \rho_{0}, z, z_{0}\right)=\frac{4(-1)^{l} \cos \left(l \alpha_{0}\right)}{\sqrt{\left(\rho+\rho_{0}\right)^{2}+\left(z-z_{0}\right)^{2}}} \sum_{k=0}^{l} \frac{(-1)^{k} 4^{k} l}{k+l} C_{l+k}^{2 k} \int_{0}^{\frac{\pi}{2}} \frac{\sin ^{2 k} \theta d \theta}{\sqrt{1-m^{2} \sin ^{2} \theta}} .
$$

The squares of the sine functions can be expanded into the following series:

$$
\sin ^{2 k} \theta=\frac{1}{m^{2 k}} \sum_{s=0}^{k}(-1)^{s} C_{k}^{s}\left(1-m^{2} \sin ^{2} \theta\right)^{s} .
$$

So we get eqn (17): 


$$
\int_{0}^{\frac{\pi}{2}} \frac{\sin ^{2 k} \theta d \theta}{\sqrt{1-m^{2} \sin ^{2} \theta}}=\frac{1}{m^{2 k}} \sum_{s=0}^{k}(-1)^{s} C_{k}^{s} \int_{0}^{\frac{\pi}{2}}\left(1-m^{2} \sin ^{2} \theta\right)^{2 s-1} 2 d \theta .
$$

Finally, in order to evaluate the integral $I_{u}\left(\rho, \rho_{0}, z, z_{0}\right)$ we used equations given in eqns (16) and (17).

In reference [15], the special elliptic integrals are introduced:

$$
E_{s}(m)=\int_{0}^{\frac{\pi}{2}}\left(1-m^{2} \sin ^{2} \theta\right)^{2 s-1} \frac{2}{2} d \theta
$$

The simplest approach for calculation of the integrals in eqn (18) is by using numeric integration, but increasing the order $s$ of integrals, so numerical error also grows. In reference [15], the recurrent equations are in use. The special elliptic integrals of order $s$ are expressed as:

$$
E_{s}(m)=Q_{s}(m) E(m)+P_{S}(m) K(m) .
$$

Here, $E(m)$ and $K(m)$ are complete elliptic integrals of the first and second kind, and $Q_{\mathrm{s}}(m)$, $P_{\mathrm{s}}(m)$ are polynomials. In previous work [15], [16], the method for high-accuracy calculations of complete elliptic integrals of the first and second kind is developed using the well-known arithmetic-geometric mean (AGM), Gauss' technique [17].

The analogical procedure is elaborated here for evaluating the integral $I_{q}\left(\rho, \rho_{0}, z, z_{0}\right)$. Here the relation for eqn (19) is also obtained, but with other polynomials $Q_{\mathrm{s}}(m)$ and $P_{\mathrm{s}}(m)$.

After calculating the inner integrals $I_{u}\left(\rho, \rho_{0}, z, z_{0}\right)$ and $I_{q}\left(\rho, \rho_{0}, z, z_{0}\right)$ in eqn (13), the boundary element method (BEM) with constant approximation of densities was applied to obtain unknown functions using a special approach, to calculate the outer integrals with logarithmical singularities [2], [5].

\section{NUMERICAL SIMULATIONS}

\subsection{Benchmark test}

For testifying the proposed numerical algorithm with qualified evaluation of inner integrals the liquid frequencies in the rigid cylindrical shell are compared with R. Ibrahim's analytical solutions (eqn 20), as seen in previously published work [18]:

$$
\frac{\chi_{k}^{2}}{g}=\frac{\mu_{k}}{R} \tanh \left(\mu_{k} \frac{H}{R}\right), k=1,2, . . ; \varphi_{k}=J_{l}\left(\frac{\mu_{k}}{R} r\right) \cosh \left(\frac{\mu_{k}}{R} z\right) \cosh ^{-1}\left(\frac{\mu_{k}}{R} H\right) .
$$

Here, $R$ is the shell radius, $H$ is its height, $\mu_{k}$ values are roots of the equation $J_{l}^{\prime}(x)=0$, the $J_{l}(x)$ is a Bessel function of the first kind; and $\chi_{k}, \varphi_{k}$ are the frequencies and modes of liquid sloshing within the rigid cylindrical shell. The numerical solutions are obtained by using the BEM as it was described beforehand, as well as by the method developed in reference [8].

Suppose that $R=1 \mathrm{~m}$ and $H=1 \mathrm{~m}$. Table 1 provides the numerical values of the natural frequencies of liquid sloshing for $l=1$, obtained by the proposed numerical method, the 
method developed in reference [8], and the analytical solution for eqn (20). Thus, Table 1 results testify to the convergence of our proposed BEM and its effectiveness. Here, in both numerical methods, we considered equal numbers of $N=150$ for the boundary elements along $\Gamma$.

Table 1: Slosh frequency parameters $\chi_{n}^{2} / g$ of a fluid-filled rigid cylindrical shell, using different solving methods.

\begin{tabular}{|l|c|c|c|c|c|}
\hline Method & $n=1$ & $n=2$ & $n=3$ & $n=4$ & $n=5$ \\
\hline$[8]$ & 1.6590 & 5.3301 & 8.5385 & 11.7071 & 14.8684 \\
\hline Proposed BEM & 1.6573 & 5.3293 & 8.5363 & 11.7060 & 14.8635 \\
\hline Analytical solution (20) & 1.6573 & 5.3293 & 8.5363 & 11.7060 & 14.8635 \\
\hline
\end{tabular}

The results obtained by both numerical methods were very close to the analytical ones. It should be noted that the results obtained by our new variant of BEM, with qualified evaluation of inner integrals, are essentially more accurate.

\subsection{Vibrations of elastic fluid-filled truncated conical shells}

We also provide estimation of natural modes and frequencies of an elastic truncated cone coupled with liquid sloshing. In Table 2, an elastic conical tank with clamped-free edges was considered, supposing that $R=0.5 \mathrm{~m}, H=1 \mathrm{~m}$ and $\theta=\pi / 4$. Results obtained were for seven wave numbers, $l=\overline{0.6}$ with $k=\overline{1.4}$. The frequencies of liquid sloshing, vibrations of empty and liquid-filled tanks are obtained. The results of numerical simulations are given in Table 2 .

For these numerical simulations, three basic systems were built, similar to previous studies [6]. The elastic empty shell modes are first. The displacements in the coupled problem are considered as the linear combinations of the empty elastic shell natural modes. Free vibration modes and frequencies for the liquid-filled elastic shell, without gravity effects, are defined at the second step. These are the second system of basic functions. The third system consists of sloshing modes, including gravity effects. The numerical simulation is accomplished using the developed one-dimensional BEM with qualified evaluation of the inner integrals.

Hereinafter, in numerical simulation, the shell thickness and Poisson's ratio were taken as $h / R=0.01$ and $v=0.3$, Young's modulus was $E=2.11 \times 10^{6} \mathrm{MPa}$, and densities for shells and liquid are $\rho_{s}=8,000 \mathrm{~kg} / \mathrm{m}^{3}$ and $\rho_{l}=1,000 \mathrm{~kg} / \mathrm{m}^{3}$, respectively.

In this case, the mutual influence of sloshing and elastic shell vibrations is negligible. The separation of frequency spectra for the liquid-filled elastic cone tank and the liquid sloshing within the rigid tank was observed.

The conclusions following numerical simulations are interesting: First, the lowest frequency here belongs to the axisymmetric mode with dominant bottom vibrations. The axisymmetric modes for the bottom and the walls of the truncated conical tanks are demonstrated in Figs 2 and 3. 
Table 2: Frequencies in elastic truncated conical shell, in Hertz (Hz).

\begin{tabular}{|c|c|c|c|c|}
\hline \multirow{2}{*}{$n$} & \multirow{2}{*}{$m$} & \multicolumn{3}{|c|}{ Frequencies } \\
\hline & & Sloshing & Empty & Fluid-filled \\
\hline \multirow{4}{*}{0} & 1 & 5.836 & 101.07 & 41.67 \\
\hline & 2 & 8.300 & 393.49 & 214.06 \\
\hline & 3 & 9.997 & 559.52 & 257.91 \\
\hline & 4 & 11.443 & 675.88 & 471.43 \\
\hline \multirow{4}{*}{1} & 1 & 3.659 & 210.34 & 113.56 \\
\hline & 2 & 7.001 & 327.90 & 126.64 \\
\hline & 3 & 8.979 & 601.83 & 425.00 \\
\hline & 4 & 10.577 & 649.99 & 438.22 \\
\hline \multirow{4}{*}{2} & 1 & 4.819 & 193.05 & 96.57 \\
\hline & 2 & 7.897 & 345.07 & 224.03 \\
\hline & 3 & 9.729 & 605.43 & 346.52 \\
\hline & 4 & 11.236 & 764.39 & 500.66 \\
\hline \multirow{4}{*}{3} & 1 & 5.707 & 128.30 & 64.217 \\
\hline & 2 & 8.661 & 504.88 & 281.69 \\
\hline & 3 & 10.397 & 519.84 & 327.95 \\
\hline & 4 & 11.837 & 723.61 & 474.97 \\
\hline \multirow{4}{*}{4} & 1 & 6.460 & 100.89 & 58.200 \\
\hline & 2 & 9.340 & 436.93 & 467.25 \\
\hline & 3 & 11.005 & 689.26 & 265.86 \\
\hline & 4 & 12.394 & 693.20 & 506.67 \\
\hline \multirow{4}{*}{5} & 1 & 7.1288 & 101.85 & 56.908 \\
\hline & 2 & 9.9581 & 385.15 & 232.28 \\
\hline & 3 & 11.568 & 671.56 & 452.47 \\
\hline & 4 & 12.915 & 897.75 & 686.67 \\
\hline \multirow{4}{*}{6} & 1 & 7.736 & 123.20 & 78.861 \\
\hline & 2 & 10.529 & 368.32 & 241.18 \\
\hline & 3 & 12.094 & 663.03 & 458.88 \\
\hline & 4 & 13.406 & 952.83 & 688.34 \\
\hline
\end{tabular}

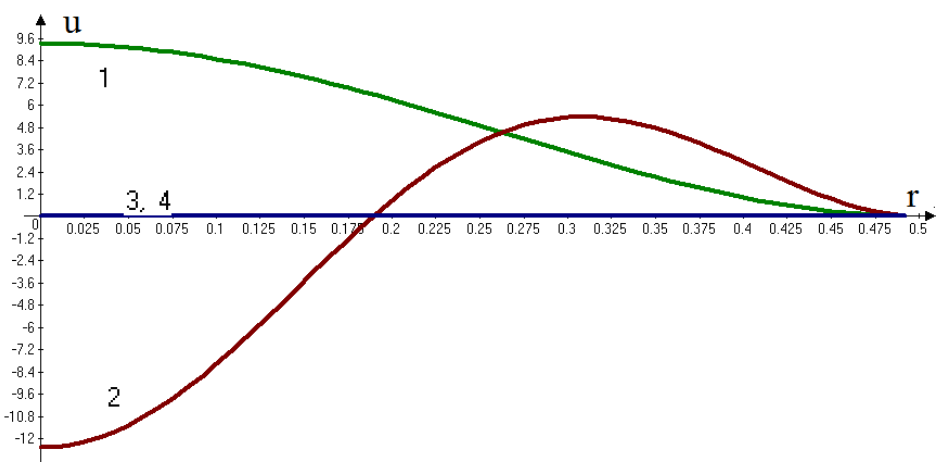

Figure 2: Axisymmetric modes of tank bottom vibrations. 


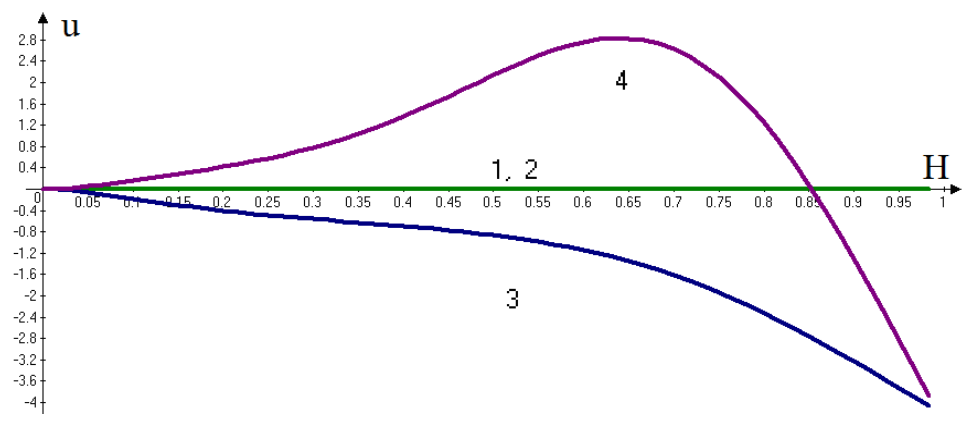

Figure 3: Axisymmetric modes of tank wall vibrations.

Hereinafter, the numbers $1,2,3$ and 4 correspond to the vibration numbers for $k$ with $l=0$. Figs 2 and 3 demonstrate different behaviour for the bottom and for the shell walls' vibrations. One can observe that in this case, the bottom and wall vibrations do not affect each other. Note that the frequency $\omega=41.67 \mathrm{~Hz}$ is the lowest one for vibrations of the liquid-filled elastic conical shell with elastic bottom. It corresponds to $l=0$ and $k=1$. If conical shells with rigid bottoms are considered, then the lowest frequency occurs at $l=4$ and $k=1$ for the empty shell; and $l=5$ and $k=1$ for the fluid-filled shell. Fig. 4 demonstrates that modes corresponding to the bottom vibrations are $l=5$ and $k=1$.

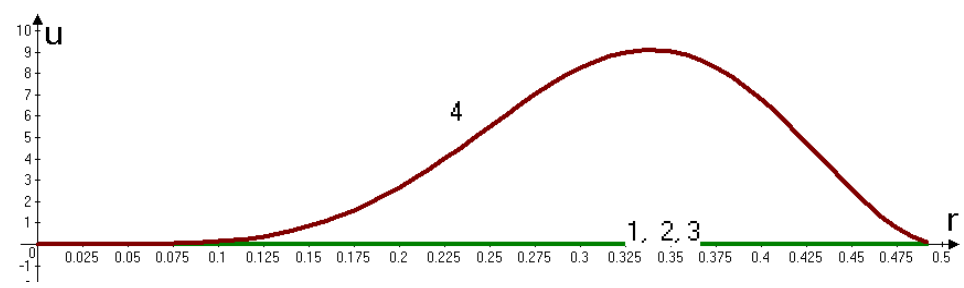

Figure 4: Modes of tank bottom vibrations given $l=5$ and $k=1$.

Fig. 5 demonstrates the wall vibration modes for $l=5$ and $k=1$. The lowest frequency here responds to the wall vibrations. Thus, if the truncated elastic conical tank with a rigid bottom is considered, then the lowest frequency does not correspond to the axisymmetric mode.

Analysis of Figs 2-5 should lead to an understanding that at low wave numbers, the dominant modes of truncated elastic cone vibrations correspond to its bottom, yet with increasing the number of nodal diameters, the tank's wall vibrations become dominant.

Fig. 6 shows the modes of lowest frequencies for liquid sloshing in the rigid tank (left); plus for the elastic conical tank with the rigid bottom (right).

If the bottom deformation is neglected, then the lowest frequency of elastic fluid-filled shell would be missed.

The frequencies $(\omega)$ near $100 \mathrm{~Hz}$ were considered as being most dangerous for empty shells. Our results, provided in Table 2, testify to it. For example, a $\omega=101.07 \mathrm{~Hz}$ corresponds to $l=0$ and $k=1$; and a $\omega=100.89 \mathrm{~Hz}$ corresponds to an $l=4$ and $k=1$; plus a $\omega=101.86 \mathrm{~Hz}$ corresponds to $\underline{l}=5$ and $k=1$. 


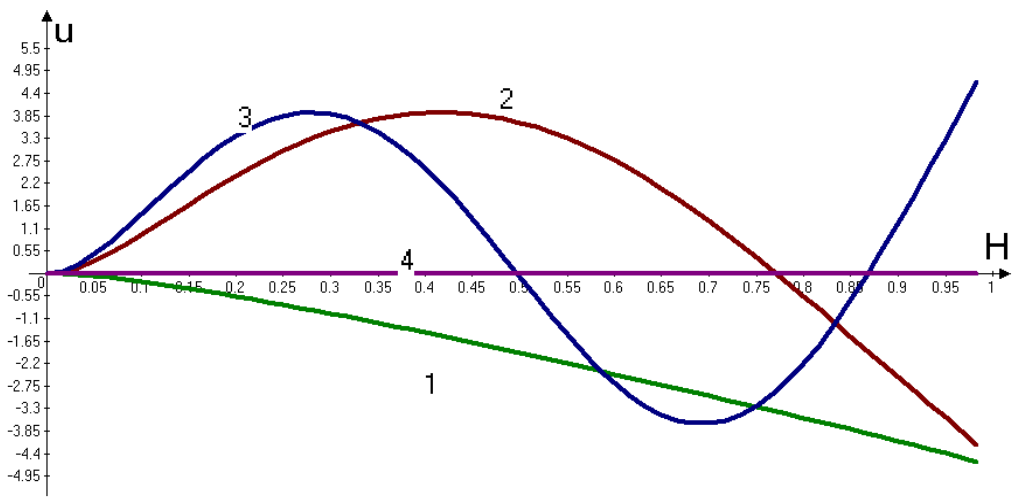

Figure 5: Modes of tank wall vibrations for $l=5$ and $k=1$.
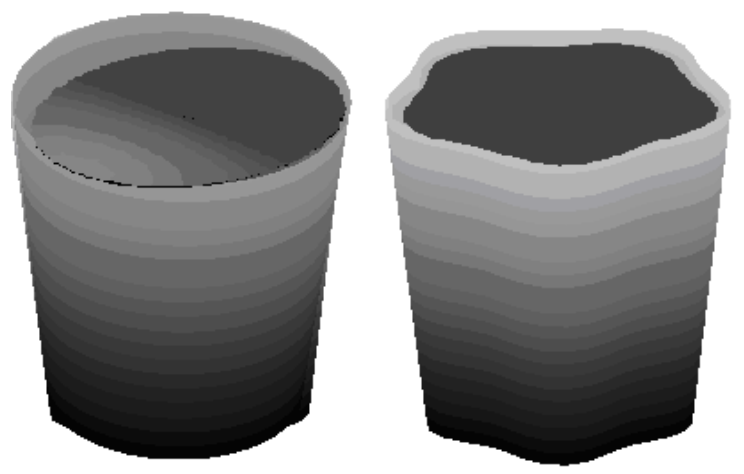

Figure 6: Modes of lowest frequencies.

It is also important to note that the lowest frequencies of the empty and liquid-filled tanks corresponded to different circumferential wave numbers.

The frequencies of liquid-filled tank vibrations are drastically different from the frequencies of empty tanks; however, with increasing the wave number, this difference became gradually smaller.

\section{CONCLUSIONS}

We propose a new variant of BEM that solves the Laplace equation with axisymmetric calculation domain, giving periodic functions under boundary conditions. The shape of the calculation domain allowed us to reduce surface integral equations down to onedimensional ones. We introduced special elliptic integrals and developed an advanced numerical method for their evaluation. We analysed the natural vibrations for truncated elastic shell interactions with internal liquid sloshing/rotating within shell forms. Coupled one-dimensional finite and boundary element methods were used. The vibration analysis included several steps. The numerical simulation was accomplished using the developed one-dimensional BEM with qualified evaluation of inner integrals. The developed method essentially reduces computer time for numerical analysis and produces new qualitative 
possibilities in advanced computational modelling of the dynamic characteristics of liquidfilled elastic shell structures. We demonstrated the differences in dynamic characteristics between elastic truncated shells with rigid and elastic bottoms. Our results can be considered as a basis for further research in the dynamic behaviour of structures subjected to intensive loading and fluid interactions. It should be noted that our interpretation and understanding of the dynamic processes found in elastic shell structures subjected to the actions of flowing fluids is far from completion and requires additional research.

\section{ACKNOWLEDGEMENTS}

We gratefully acknowledge financial support for our project from the Ministry of Education and Science of Ukraine. The authors would also like to thank our foreign collaborator, Professor Alexander Cheng, at the University of Mississippi, USA, for his constant support and interest to our research.

\section{REFERENCES}

[1] Sadaba, S., Herraez, M., Naya, F., Gonzalez C., Llorca, J. \& Lopes, C.S., Specialpurpose elements to impose periodic boundary conditions for multiscale computational homogenization of composite materials with the explicit finite element method. Composite Structures, 193, pp. 1-24, 2018.

DOI: $10.1016 /$ j.compstruct.2018.10.037.

[2] Gnitko, V., Degtyariov, K., Karaiev, A. \& Strelnikova, E., Multi-domain boundary element method for axisymmetric problems in potential theory and linear isotropic elasticity. WIT Transactions in Engineering Sciences, vol. 122, WIT Press: Southampton and Boston, pp. 13-25, 2019. DOI: 10.2495/BE410021.

[3] Rao, L.B. \& Rao, C.K., Free vibration of circular plates with elastic edge support and resting on an elastic foundation. International Journal of Acoustics and Vibration, 17(4), pp. 204-207, 2012.

[4] Liu, C., Yang, M., Han, H. \& Yue, W., Numerical simulation of fracture characteristics of jointed rock masses under blasting load. Engineering Computation, 36, pp. 1835-1851, 2019.

[5] Gnitko, V., Marchenko, U., Naumenko, V. \& Strelnikova, E., Forced vibrations of tanks partially filled with the liquid under seismic load. WIT Transactions on Modelling and Simulation, vol. 52, WIT Press: Southampton and Boston, pp. 285296, 2011. DOI: 10.2495/BE110251.

[6] Strelnikova, E., Choudhary, N., Kriutchenko, D., Gnitko, V. \& Tonkonozhenko, A., Liquid vibrations in circular cylindrical tanks with and without baffles under horizontal and vertical excitations. Engineering Analysis with Boundary Elements, 120, pp. 13-27, 2020. DOI: 10.1016/j.enganabound.2020.07.024.

[7] Misura, S., Smetankina, N. \& Misiura, I., Optimal design of the cyclically symmetrical structure under static load. Integrated Computer Technologies in Mechanical Engineering 2020, Springer: Cham, pp. 256-266, 2020.

DOI: 10.1007/978-3-030-66717-7_21.

[8] Gnitko, V., Degtyariov, K., Karaiev, A. \& Strelnikova, E., Singular boundary method in a free vibration analysis of compound liquid-filled shells. WIT Transactions on Engineering Sciences, vol. 126, WIT Press: Southampton and Boston, pp. 189-200, 2019. DOI: $10.2495 / B E 420171$.

[9] Smadi, M., Arqub, O.A. \& Ajou, A., A numerical iterative method for solving systems of first-order periodic boundary value problems. Journal of Applied Mathematics, 2014, 10 p., 2014. DOI: 10.1155/2014/135465. 
[10] Lakshmikantan, V., Periodic boundary conditions of first and second order differential equations. Journal of Applied Mathematics and Simulations, 2(3), pp. 131-139, 1989.

[11] Tomeček, J., Dirichlet boundary value problem for differential equation with $\phi$ Laplacian and state-dependent impulses. Mathematica Slovaca, 67(2), pp. 483-494, 2017. DOI: $10.1515 / \mathrm{ms}-2016-0283$.

[12] Brebbia, C.A., Telles, J.C.F. \& Wrobel, L.C., Boundary Element Techniques, Springer-Verlag: Berlin and New York, 1984.

[13] Gavrilyuk, I., Lukovsky, I., Trotsenko, Y. \& Timokha, A., Sloshing in a vertical circular cylindrical tank with an annular baffle. Part 1: Linear fundamental solutions. Journal of Engineering Mathematics, 54, 2006, pp. 71-88.

[14] Egorychev, G.P., Method of coefficients: An algebraic characterization and recent applications. Advances in Combinatorial Mathematics: Proceedings of the Waterloo Workshop in Computer Algebra 2008, Springer, Devoted to the 70th birthday of G. Egorychev, pp. 1-30, 2009.

[15] Karaiev, A. \& Strelnikova, E., Axisymmetric polyharmonic spline approximation in the dual reciprocity method. Zeitschrift für Angewandte Mathematik und Mechanik, 101, p. e201800339, 2021. DOI: 10.1002/zamm.201800339.

[16] Zheng, L., Gaofeng, W., Zhiming, W. \& Jinwei, Q., The meshfree analysis of geometrically nonlinear problem based on radial basis reproducing kernel particle method. International Journal of Applied Mechanics, 12(4), p. 2050044, 2020. DOI: $10.1142 / \mathrm{S} 1758825120500441$.

[17] Cox, D.A., The arithmetic-geometric mean of gauss. L'Enseignement Mathématique, 30, pp. 275-330, 1984.

[18] Ibrahim, R.A., Liquid Sloshing Dynamics. Theory and Applications. Cambridge University Press, 972 pp., 2005. 